\title{
Biobrane dressing for paediatric burns in Singapore: a retrospective review
}

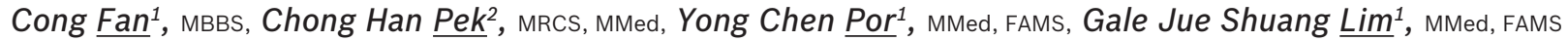

\begin{abstract}
INTRODUCTION The ideal burn dressing for children should aim to alleviate pain, decrease length of hospital stay and minimise complications such as conversion and infection. The current literature is still inconclusive with regard to the gold standard burn dressing for the paediatric population.

METHODS We retrospectively reviewed children with superficial partial thickness burns admitted to our paediatric burns unit from January 2014 to April 2015. A total of 30 patients were included in our study, of whom 13 had Biobrane ${ }^{\circledR}$ dressing. The remaining 17 patients were treated with conventional silver foam dressing (i.e. Biatain ${ }^{\circledR} \mathrm{Ag}$ ) and served as matched controls. Long-term follow-up scar evaluation was carried out at an average interval of two years after injury. RESULTS In the Biobrane group, the length of hospital stay was significantly shorter (Biobrane vs. silver foam: $4.76 \pm$ 2.64 days vs. $8.88 \pm 5.09$ days; $p=0.01$ ) and the infection rate was significantly lower (Biobrane vs. silver foam: $0 \%$ vs. $35.3 \% ; p=0.02$ ). The Biobrane group had no hypergranulation or wound infection and did not require skin grafting. Longterm follow-up scar evaluation did not reveal any statistical difference between the patient groups at the two-year interval. CONCLUSION Paediatric patients with partial thickness burns treated with Biobrane dressing had shorter hospital stay and lower incidence of infection compared to those treated with conventional silver foam dressing. Biobrane and silver foam dressings did not demonstrate any significant difference in terms of long-term scar outcomes over an average follow-up duration of two years.
\end{abstract}

Keywords: Biobrane, paediatric burns, silver dressing, skin substitutes, superficial partial thickness burns

\section{INTRODUCTION}

The paediatric burns unit at KK Women's and Children's Hospital, Singapore, sees an average of 350 attendances a year for paediatric burns. A majority (82\%) of these patients have scald injuries and are 1-2 years of age. Young toddlers are at a higher risk of deeper burns or burn conversion (the interval worsening or deepening of the depth of burn wounds over time) due to their thinner dermis compared to adults. ${ }^{(1)}$ The conventional management principle for burn injuries includes adequate resuscitation and early debridement of non-viable tissue, followed by skin grafting. Early scrub down and dressing of superficial partial thickness burns can reduce bacterial colonisation, potentially reducing burn conversion and promoting uncomplicated healing.

Frequent dressing changes required for burn injuries are often painful and a source of great anxiety for the child and the parents. Modern burn dressings aim to not only provide good wound coverage but also reduce the frequency of dressing changes needed. ${ }^{(2,3)}$ As the paediatric population is prone to hypertrophic scarring after burns, rapid epithelialisation is desirable to reduce the risk of scarring.

For many years, prior to the introduction of Biobrane ${ }^{\circledR}$ (UDL Laboratories, Rockford, IL, USA), acute partial thickness burns were all managed with conventional silver-impregnated dressings, at most times producing satisfactory results. Some examples of the commonly used silver-impregnated dressings are Mepilex Ag (Mölnlycke Health Care AB, Gamlestadsvägen, Gothenburg,
Sweden), Biatain ${ }^{\circledR}$ Ag (Coloplast, Mount Waverley, Victoria, Australia) and the nanocrystalline silver-containing Acticoat ${ }^{\mathrm{TM}}$ (Smith \& Nephew, Hull, UK). The use of silver sulfadiazine cream for large burn wounds has fallen out of favour due to the need for very frequent and painful application as well as reports of delayed wound healing. ${ }^{(1,4-7)}$

Biobrane is a bilaminate biosynthetic material comprising a semipermeable silicone membrane bonded to a layer of nylon fabric mesh that is coated with a layer of Type I collagen of porcine origin. Early application of the Biobrane dressing has been increasingly advocated for patients with partial thickness burn injuries, ${ }^{(8-15)}$ as it possesses many characteristics of optimal burns dressing - appropriate wound adhesion, good vapour transmission, flexibility, elasticity and sufficient transparency for wound observation. ${ }^{(9,12)}$ Upon application, Biobrane is designed to adhere to superficial partial thickness burns and provide definitive coverage for such burn injuries. Once firmly adhered, Biobrane provides a barrier to pathogens and reduces exudate production from burn wounds. Reduced exudate production lowers fluid loss and, in turn, helps to prevent dehydration in the paediatric burns patient.

Despite its apparent popularity, Biobrane was not universally accepted as the optimal burns dressing for partial thickness burns. Some reasons stated in the literature include higher costs, risk of infection and the requirement of general anaesthesia for its application..$^{(12,16,17)}$ The indication for its use was limited to

${ }^{1}$ Department of Plastic, Reconstructive and Aesthetic Surgery, KK Women's and Children's Hospital, ${ }^{2}$ Plastic, Reconstructive and Aesthetic Surgery Section, Department of General Surgery, Tan Tock Seng Hospital, Singapore

Correspondence: Dr Pek Chong Han, Plastic, Reconstructive and Aesthetic Surgery Section, Department of General Surgery, Tan Tock Seng Hospital, 11 Jalan Tan Tock Seng, Singapore 308433. pekch@rocketmail.com 


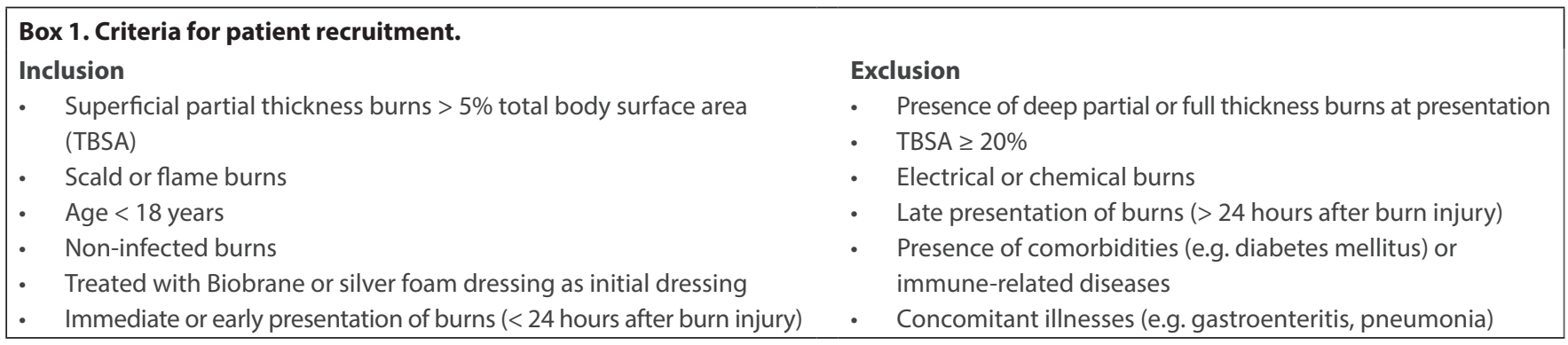

superficial partial thickness burns and it was not considered appropriate for deeper burns. In our practice, we also occasionally encounter Muslim patients who reject the use of Biobrane due to its porcine origins. This may possibly explain the general lack of literature on the use of Biobrane for paediatric burns patients in the Asian context.

Biobrane was reintroduced to our paediatric burns unit in 2014 after good results were reported from the adult burns centre at Singapore General Hospital, Singapore. ${ }^{(18)}$ Since then, Biobrane has routinely been offered as the first-line burns dressing to paediatric patients with superficial partial thickness burns of more than $5 \%$ of total body surface area (TBSA) who present within 24 hours of the injury. This study aimed to compare the results of Biobrane dressing with conventional silver foam dressing (i.e. Biatain Ag) for primary coverage of acute superficial partial thickness burns.

\section{METHODS}

A retrospective review was performed of paediatric burns patients admitted to our paediatric burns unit from January 2014 to April 2015. Data collected from the patients' medical records included age, gender, extent of burn injury (in terms of TBSA), number of inpatient and outpatient dressing changes, length of hospital stay, time to full epithelialisation, wound infection rate, conversion rate and wound hypergranulation rate.

We aimed to review the use of Biobrane dressing compared with conventional silver foam dressing for paediatric patients with superficial partial thickness burns. We hypothesised that Biobrane dressing would provide superior short-term outcomes for paediatric burns patients with respect to: (a) shorter hospitalisation stay; and (b) reduced complications, such as wound infection, burn conversion and secondary surgery (i.e. skin graft). As a secondary long-term outcome, we also hypothesised that Biobrane would lead to superior long-term scar outcomes.

A total of 30 patients who satisfied the inclusion and exclusion criteria (Box 1) were included in the study. Of these patients, 13 were treated with Biobrane dressings and conventional silver foam dressing was used for the remaining 17 patients. Biobrane was offered as the first-line burns dressing to all patients with acute superficial partial thickness burns of more than 5\% TBSA presenting to the hospital within 24 hours of injury. However, it was not offered to those with extensive deep dermal or full thickness burns, or for patients with chemical or electrical burns. A key reason for patients not receiving Biobrane dressing was patient and/or family refusal. Patients who refused

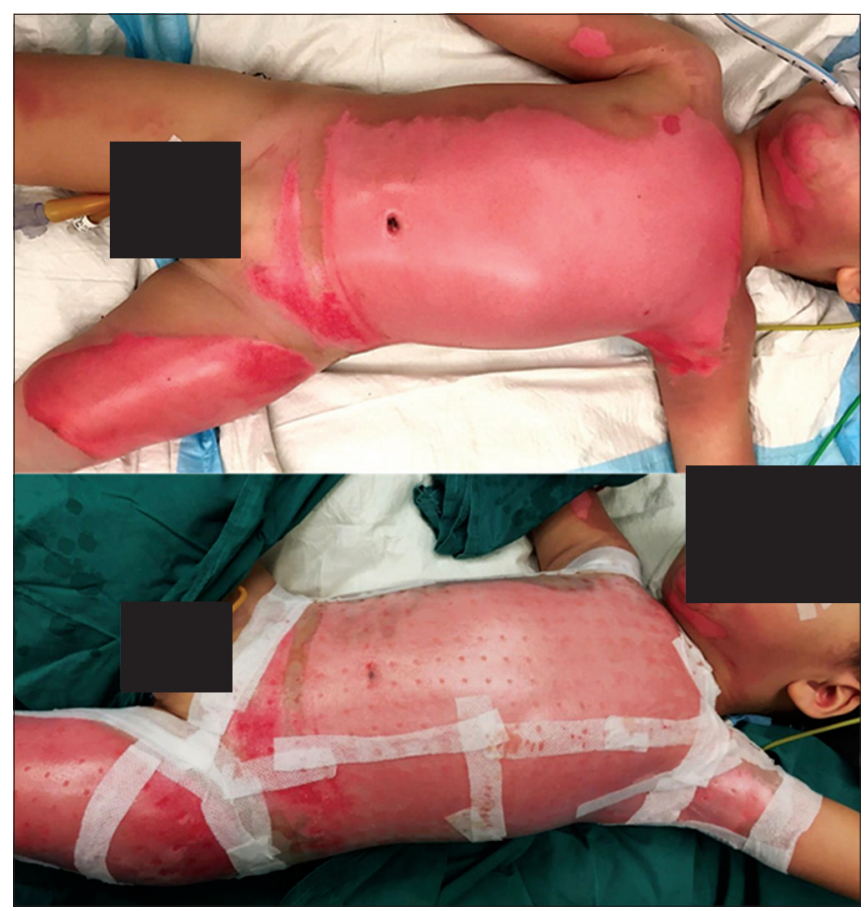

Fig. 1 Photographs show application of Biobrane dressing on a child with superficial partial thickness burns. Biobrane dressing was trimmed to size and applied onto the wound under mild tension to improve adherence of the dressing to the wound. It was then fixed to adjacent healthy skin with skin staples or absorbable suture at key points. The borders of the dressing were further reinforced using adhesive tape. Acticoat or dilute iodine gauze was applied as secondary dressing.

Biobrane were managed using conventional silver foam dressing (i.e. Biatain Ag).

We selected 17 matched controls from the silver foam dressing group based on patient demographics, such as age, gender, percentage of TBSA, and depth and mechanism of burns (all patients had superficial partial thickness burns on presentation). Patient demographics were comparable in the two groups.

All 30 patients had early scrub-down and wound coverage with either Biobrane or conventional silver foam dressing under sterile conditions, with general anaesthesia support. During application, Biobrane was trimmed to size and applied onto the wound under mild tension to improve contact with the wound. It was then fixed to the adjacent healthy skin with staples or absorbable sutures (polyglactin 6/0) at key points. The borders of the dressing were reinforced with adhesive tape (Fig. 1). Dilute iodine $(0.25 \%)$ gauze, with overlying dry gauze, was used as a secondary dressing. The wounds were examined under sterile 
Table I. Patient demographics.

\begin{tabular}{|llll|}
\hline Variable & \multicolumn{1}{c}{ No. $(\%) /$ mean \pm standard deviation } & p-value \\
\cline { 2 - 3 } & Biobrane $(\mathbf{n = 1 3 )}$ & Silver foam $(\mathbf{n = 1 7 )}$ & 0.70 \\
\hline Age $(\mathbf{y r})$ & $3.64 \pm 4.01$ & $3.13 \pm 3.14$ & 0.46 \\
\hline Gender & & & $6(35.3)$ \\
\hline Female & $7(53.8)$ & $11(64.7)$ & 0.12 \\
\hline Male & $6(46.2)$ & $11.68 \pm 4.67$ & 0.19 \\
\hline Total body surface area (\%) & $8.96 \pm 4.36$ & $19.53 \pm 13.02$ & 0.18 \\
\hline Time from injury to coverage $(\mathbf{h r})$ & $14.42 \pm 5.09$ & & $17(100.0)$ \\
\hline Mechanism of burn & & $0(0)$ & \\
\hline Scald & $11(84.6)$ & & \\
\hline Flame & $2(15.4)$ & & \\
\hline
\end{tabular}

conditions on Postoperative Day 2 or 3 to determine the extent of Biobrane adherence and burn conversion, if any. Staples or sutures were removed, as necessary, at this stage. If an area of nonadherence was noted, that patch of Biobrane was trimmed off. In the case of a purulent collection, the Biobrane dressing was removed in the area and a tissue culture was performed. The resulting exposed portion was covered using silver foam dressing. The external dressings were changed after wound inspection. Wounds were reassessed every $3-5$ days afterwards, and subsequently exposed once epithelialisation was achieved.

In the control group, burns were scrubbed down under sterile conditions and conventional silver foam dressing applied and reinforced with adhesive tape. The wounds were reassessed in a similar time frame of about three days postoperatively. The dressings were changed about twice weekly in the outpatient setting thereafter. The wounds were exposed once fully epithelised. For both patient groups, all other aspects of burns management were standardised to our unit's burn care protocol, including initial fluid resuscitation, inpatient nursing care, postoperative pain relief, nutritional supplementation and outpatient care.

Infection was defined as any purulent collection with positive bacterial growth on tissue culture. Time to full epithelialisation was defined as the number of days from the burn injury to complete epithelialisation of the whole wound, without requiring any further wound dressing. Wound conversion was defined as the interval worsening or deepening of the depth of the burn wounds upon subsequent wound inspections. All of the above assessments were done by the attending paediatric burns surgeon in charge.

A long-term scar evaluation was conducted in November 2016. As most of our patients were no longer on outpatient follow-up with us after their scars had matured, scar evaluation was conducted via telephone interview using the Patient Scar Assessment Scale (PSAS) from the Patient and Observer Scar Assessment Scale. ${ }^{(19)}$ The scars were evaluated based on individual characteristics, such as scar pain, itch, colour, thickness, stiffness and irregularity, and graded individually on a numeric severity scale of $0-10$.

Data was analysed using IBM SPSS Statistics for Windows version 22.0 (IBM Corp, New York, USA). Biostatistical data was presented as mean \pm standard deviation. Student's t-test was performed for variables, and categorical variables were compared using Fisher's exact test. All testing was two-tailed and $p<0.05$ was considered to be statistically significant.

\section{RESULTS}

Patients included in our study were mostly preschool children (Biobrane vs. silver foam: mean age $3.64 \pm 4.01$ years vs. $3.13 \pm$ 3.14 years). All had sustained superficial partial thickness burns. Most $(n=28,93.3 \%)$ patients had scald burns; only $2(6.7 \%)$ patients had flame burns (Table I). There was no statistical difference in mean time from injury to wound coverage between the Biobrane and silver foam dressing groups (Biobrane vs. silver foam: $14.42 \pm 5.09$ hours vs. $19.53 \pm 13.02$ hours; $p=0.19$ ).

Compared with the silver foam group, the Biobrane group had significantly shorter length of hospital stay (Biobrane vs. silver foam: $4.76 \pm 2.64$ days vs. $8.88 \pm 5.09$ days, $p=0.01$ ) and decreased infection rate (Biobrane vs. silver foam: $0 \%$ vs. $35.3 \%, p=0.02$; Table II). In the Biobrane group, no wound hypergranulation was observed and no skin graft was required. In contrast, in the silver foam group, hypergranulation was noted in $3(17.6 \%)$ patients and $4(23.5 \%)$ out of 17 patients required split-thickness skin graft (STSG) for areas that had converted to deep partial thickness burns. There was no significant difference between the Biobrane and silver foam dressing groups with respect to the total frequency of dressing change or time to full epithelialisation.

In our study, the Biobrane group had a lower burn conversion rate: $3(23.1 \%)$ out of 13 patients were noted to have small, scattered areas of burn conversion after application of Biobrane, whereas 9 (52.9\%) out of 17 patients from the silver foam group had areas of burn conversion. However, this difference in the burn conversion rate did not reach statistical significance, likely due to the lack of patient numbers. Burn conversion in the Biobrane group presented as areas where the Biobrane had become nonadherent, and the burn wound blanched less readily or did not blanch. The three patients from the Biobrane group with limited burn conversion went on to heal on their own without requiring skin grafts. In contrast, the converted areas in the silver foam group were larger and sometimes deeper areas, hence requiring STSG. 
Table II. Clinical comparison of patients.

\begin{tabular}{|c|c|c|c|}
\hline \multirow[t]{2}{*}{ Variable } & \multicolumn{2}{|c|}{ No. (\%) } & \multirow[t]{2}{*}{ p-value } \\
\hline & Biobrane $(n=13)$ & Silver foam $(n=17)$ & \\
\hline \multicolumn{4}{|l|}{ Frequency of dressing change* } \\
\hline Operation theatre & $2.30 \pm 0.82$ & $2.94 \pm 1.59$ & 0.20 \\
\hline Clinic & $2.64 \pm 2.14$ & $2.06 \pm 1.11$ & 0.58 \\
\hline Time to heal (day)* & $19.54 \pm 10.14$ & $18.35 \pm 8.98$ & 0.74 \\
\hline STSG requirement & $0(0)$ & $4(23.5)$ & 0.11 \\
\hline Infection rate & $0(0)$ & $6(35.3)$ & $0.02^{\dagger}$ \\
\hline Hypergranulation rate & $0(0)$ & $3(17.6)$ & 0.24 \\
\hline Conversion rate & $3(23.1)$ & $9(52.9)$ & 0.14 \\
\hline
\end{tabular}

*Data presented as mean \pm standard deviation. $\mathrm{tp}<0.05$ was considered statistically significant. STSG: split-thickness skin graft

Table III. Long-term Patient Scar Assessment Scale outcomes of patients.

\begin{tabular}{|c|c|c|c|}
\hline \multirow[t]{2}{*}{ Variable } & \multicolumn{2}{|c|}{$\%$} & \multirow[t]{2}{*}{ p-value } \\
\hline & Biobrane $(n=10)$ & Silver foam $(n=14)$ & \\
\hline Time interval after burn injury at follow-up (mth)* & $23.00 \pm 2.48$ & $25.28 \pm 3.26$ & 0.08 \\
\hline Follow-up rate (\%) & 76.9 & 82.4 & NA \\
\hline \multicolumn{4}{|l|}{ Outcomes } \\
\hline Pain & 0 & 0 & NA \\
\hline Score* & 0 & 0 & NA \\
\hline Itchiness & 20.0 & 21.4 & 1.00 \\
\hline Score* & $0.40 \pm 0.80$ & $0.36 \pm 0.72$ & 0.90 \\
\hline Colour difference & 80.0 & 92.8 & 0.55 \\
\hline Score* & $1.60 \pm 1.11$ & $2.00 \pm 1.85$ & 0.55 \\
\hline Thickness & 40.0 & 42.8 & 1.00 \\
\hline Score $e^{*}$ & $1.00 \pm 1.26$ & $1.00 \pm 1.46$ & 1.00 \\
\hline Stiffness & 20.0 & 50.0 & 0.21 \\
\hline Score* & $0.40 \pm 0.92$ & $1.21 \pm 1.82$ & 0.21 \\
\hline Irregularity & 20.0 & 21.4 & 1.00 \\
\hline Score* & $0.30 \pm 0.64$ & $0.50 \pm 1.12$ & 0.62 \\
\hline
\end{tabular}

${ }^{*}$ Data presented as mean \pm standard deviation. NA: not available

For long-term scar evaluation, a telephone interview was conducted at an average interval of two years post injury (Biobrane vs. silver foam: $23.00 \pm 2.48$ months vs. $25.28 \pm$ 3.26 months; Table III). The percentage of patients who were lost to follow-up was similar in both groups. Most patients who could not be contacted at two years were uncontactable due to invalid telephone numbers.

Based on the PSAS scores, we noticed that there was no statistically significant difference between the Biobrane and silver foam dressing groups in terms of the long-term scarring outcomes of scar pain, itch, colour, thickness, stiffness and irregularity. The most common complaint was scar colour difference, with both groups reporting a high incidence of colour difference (Biobrane vs. silver foam: $80.0 \%$ vs. $92.8 \%$ ). Although this was a common finding for burns scarring, the severity of scar colour difference was low (Biobrane vs. silver foam: $1.60 \pm 1.11$ vs. $2.00 \pm 1.85$, on a scale of ten). None of the patients reported painful scarring. The other findings of scar itch, thickness, stiffness and irregularity were not common and trended in favour of the Biobrane group. None of these associations reached statistical significance in our analysis, and no patients suffered from keloids or scar contractures as a result of burn injuries.

\section{DISCUSSION}

Paediatric burn demographics in Singapore mirror those of other countries, in that a majority of burns are reported among children from the preschool age group and most present with superficial partial thickness scalds. ${ }^{(10)}$ Children have a low pain threshold and suffer psychological stress in response to frequent painful dressing changes. They are also at higher risk for hypertrophic scarring. For these reasons, optimal wound dressing for children should be easy to apply, not be traumatic when removed, promote rapid epithelialisation, and reduce burn conversion and infection. Uncomplicated and rapid healing will also significantly reduce adverse scarring.

Some studies have found that the use of Biobrane resulted in less frequent dressing changes and earlier discharge to outpatient care. ${ }^{(10,11)}$ Consistent with the current literature, our study showed that patients in the Biobrane dressing group had a significantly shorter length of hospital stay when compared to those in the silver 
foam group $(4.76 \pm 2.64$ days vs. $8.88 \pm 5.09$ days, respectively). However, there have been reports of increased infection rate associated with Biobrane dressing, and hence most authors agree that it should be applied within 24-48 hours of the burn injury to reduce infection. ${ }^{(12,16,20,21)}$ In our study, no wound infection was noted in the Biobrane group, unlike other studies that reported wound infection rates of $5 \%-10.5 \% .^{(10,17)}$ In addition, the Biobrane group showed a statistically significant lower infection rate than the silver foam group (0\% vs. $35.3 \%$; $p=0.02)$.

We opine that application of the Biobrane dressing within 24 hours of injury, under aseptic conditions in the operating theatre, contributed to a lower overall complication rate and decreased the length of hospital stay. Once applied, the presence of porcine collagen within the Biobrane dressing promotes adherence of the dressing to the wound, thus reducing bacterial proliferation by minimising dead space, which results in a good antiseptic effect. ${ }^{(13)}$ This corroborates findings that have shown that Biobrane was effective in controlling bacterial growth in wounds that initially contained fewer than $10^{5}$ bacteria per gram of tissue. ${ }^{(12,21)}$ Our study did not show any statistical difference between the two groups in the frequency of wound dressing among both inpatients and outpatients, or in wound healing time.

Given these results, we were optimistic of obtaining better long-term scar outcomes in the Biobrane group, as we observed less wound infection and burn conversion, and no skin graft requirement or hypergranulation in these patients during treatment. Long-term scar evaluation showed that there was a trend towards more favourable scarring in the Biobrane group compared to patients in the silver foam group. However, the differences in long-term scar outcomes between the groups did not reach statistical significance. We hope to conduct a similar study with larger patient numbers in the future to further prove this trend.

There is no consensus on whether the Biobrane dressing actually helps to reduce the overall cost of treatment for patients with partial thickness burns. The higher cost of the dressing is a disadvantage, ${ }^{(14)}$ although some studies have reported that the use of Biobrane decreased the overall cost because sedation and dressing changes were less frequently required. ${ }^{(10,21)}$ In our local population, inpatient treatment with Biobrane dressing qualifies for government subsidies, which may improve the overall cost efficiency of treatment. Further cost-based analysis is required to further validate this hypothesis.

Due to Singapore's hot and humid equatorial climate, thick dressings are not well tolerated by active children. Even so, we observed that protective Asian parents were not keen to expose the Biobrane dressing in spite of our recommendation. Even after the burn wounds had epithelised or when the Biobrane dressing was ready to be exposed, parents often preferred to have it covered by a secondary dressing. This may have biased our results for both groups in terms of overall dressing cost and the required frequency of dressings.

While this is not important from a clinical perspective, we noted that the Biobrane dressing found lower acceptance among Muslim patient populations, largely due to its porcine origins. ${ }^{(8)}$
It is suggested that clinicians discuss this with patients' families prior to its use.

There were a few sources of bias inherent in our study. First, patients in the silver foam group had a longer time period till the surgical scrub down compared to the Biobrane group. Even though this time difference was not statistically significant, this may potentially increase the risk of wound infection in patients receiving silver foam dressing. As a protocol, however, all burn wounds admitted to our unit are temporarily covered with OPSITE dressing (Smith \& Nephew, Hull, UK) at the point of admission, while the patient awaits surgery. Next, our study was limited by lack of power and patient numbers. Finally, the retrospective nature of our study may have also contributed heavily to selection bias. We hope to conduct further prospective studies in the future with larger patient numbers to substantiate our current findings.

In conclusion, this study has shown that Biobrane is suitable for the treatment of acute superficial partial thickness burns in the paediatric population. Compared with conventional silver foam dressing, the use of Biobrane dressing for acute superficial partial thickness burns results in a shorter hospital stay, lower complication rate and less need for eventual skin grafting among paediatric patients. No significant difference was found between Biobrane and silver foam dressings in terms of long-term scar outcomes. Our results support the continued use of Biobrane as the first-line burns dressing for superficial partial thickness burns within the first 24 hours of injury. Further prospective studies with larger patient numbers and overall cost-based analysis are needed to further justify the initial financial outlay required for this dressing in burns management.

\section{REFERENCES}

1. Vloemans AF, Hermans $M H$, van der Wal MB, Liebregts J, Middelkoop E. Optimal treatment of partial thickness burns in children: a systematic review. Burns 2014; 40:177-90.

2. Delatte SJ, Evans J, Hebra A, et al. Effectiveness of beta-glucan collagen for treatment of partial-thickness burns in children. J Pediatr Surg 2001; 36:113-8.

3. Honari S. Topical therapies and antimicrobials in the management of burn wounds. Crit Care Nurs Clin North Am 2004; 16:1-11.

4. Verbelen J, Hoeksema H, Heyneman A, Pirayesh A, Monstrey S. Aquacel((囚) Ag dressing versus Acticoat ${ }^{\mathrm{TM}}$ dressing in partial thickness burns: a prospective, randomized, controlled study in 100 patients. Part 1: burn wound healing. Burns 2014; 40:416-27.

5. Atiyeh BS, Costagliola M, Hayek SN, Dibo SA. Effect of silver on burn wound infection control and healing: review of the literature. Burns 2007; 33:139-48.

6. Saba SC, Tsai R, Glat P. Clinical evaluation comparing the efficacy of Aquacel Ag Hydrofiber dressing versus petrolatum gauze with antibiotic ointment in partialthickness burns in a pediatric burn center. J Burn Care Res 2009; 30:380-5.

7. Selig HF, Lumenta DB, Giretzlehner $M$, et al. The properties of an "ideal" burn wound dressing--what do we need in daily clinical practice? Results of a worldwide online survey among burn care specialists. Burns 2012; 38:960-6.

8. Whitaker IS, Worthington S, Jivan S, Phipps A. The use of Biobrane by burn units in the United Kingdom: a national study. Burns 2007; 33:1015-20.

9. Yang JY, Tsai YC, Noordhoff MS. Clinical comparison of commercially available Biobrane preparations. Burns 1989; 15:197-203.

10. Lang EM, Eiberg CA, Brandis M, Stark GB. Biobrane in the treatment of burn and scald injuries in children. Ann Plast Surg 2005; 55:485-9.

11. Gerding RL, Emerman CL, Effron D, et al. Outpatient management of partialthickness burns: Biobrane versus 1\% silver sulfadiazine. Ann Emerg Med 1990; 19:121-4.

12. Whitaker IS, Prowse S, Potokar TS. A critical evaluation of the use of Biobrane as a biologic skin substitute: a versatile tool for the plastic and reconstructive surgeon. Ann Plast Surg 2008; 60:333-7.

13. Lal S, Barrow RE, Wolf SE, et al. Biobrane improves wound healing in burned children without increased risk of infection. Shock 2000; 14:314-9. 
14. Cassidy C, St Peter SD, Lacey S, et al. Biobrane versus duoderm for the treatmen of intermediate thickness burns in children: a prospective, randomized trial. Burns 2005; 31:890-3.

15. Wood F, Martin L, Lewis D, et al. A prospective randomised clinical pilot study to compare the effectiveness of Biobrane ${ }^{\circ}$ synthetic wound dressing, with or without autologous cell suspension, to the local standard treatment regimen in paediatric scald injuries. Burns 2012; 38:830-9.

16. Gonce S, Miskell P, Waymack JP. A comparison of Biobrane vs. homograft for coverage of contaminated burn wounds. Burns Incl Therm Inj 1988; 14:409-12.

17. Demling RH. Use of Biobrane in management of scalds. J Burn Care Rehabil 1995; $16(3$ Pt 1):329-30.
18. Cheah AK, Chong SI, Tan BK. Early Experience with Biobrane ${ }^{T M}$ in Singapore in the management of partial thickness burns. Proc Singapore Healthcare 2014; 23:196-200

19. Draaijers LI, Tempelman FR, Botman YA, et al. The patient and observer scar assessment scale: a reliable and feasible tool for scar evaluation. Plast Reconstr Surg 2004; 113:1960-7.

20. Feldman DL, Rogers A, Karpiniski RH. A prospective trial comparing Biobrane, Duoderm and xeroform for skin graft donor sites. Surg Gynaecol Obstet 1991; 173:1-5.

21. McHugh TP, Robson MC, Heggers JP, et al. Therapeutic efficacy of Biobrane in partial- and full-thickness thermal injury. Surgery 1986; 100:661-4. 